\title{
Microstructural, optical and electrical properties of Cl-doped CdTe single crystals
}

\author{
Hyojeong ChOi ${ }^{1}$, Han Soo Kim ${ }^{2}$, JoOn-Ho OH${ }^{2}$, Dong Jin Kim ${ }^{2}$, Young Soo Kim ${ }^{2}$, \\ JONG-SEO CHAI ${ }^{3}$, JANG HO HA ${ }^{2, *}$ \\ ${ }^{1}$ WCU Department of Energy Science, Sungkyunkwan University, Suwon, Republic of Korea \\ ${ }^{2}$ Korea Atomic Energy Research Institute, Jeongeup, Republic of Korea \\ ${ }^{3}$ College of Information and Communication Engineering, Sungkyunkwan University, Suwon, Republic of Korea
}

\begin{abstract}
Microstructural, optical and electrical properties of Cl-doped CdTe crystals grown by the low pressure Bridgman (LPB) method were investigated for four different doping concentrations (unintentionally doped, $4.97 \times 10^{19} \mathrm{~cm}^{-3}, 9.94 \times 10^{19} \mathrm{~cm}^{-3}$ and $1.99 \times 10^{20} \mathrm{~cm}^{-3}$ ) and three different locations within the ingots (namely, samples from top, middle and bottom positions in the order of the distance from the tip of the ingot). It was shown that $\mathrm{Cl}$ dopant suppressed the unwanted secondary (5 111$)$ crystalline orientation. Also, the average size and surface coverage of Te inclusions decreased with an increase in $\mathrm{Cl}$ doping concentration. Spectroscopic ellipsometry measurements showed that the optical quality of the Cl-doped CdTe single crystals was enhanced. The resistivity of the CdTe sample doped with $\mathrm{Cl}$ at the $1.99 \times 10^{20} \mathrm{~cm}^{-3}$ was above $10^{10} \Omega \cdot \mathrm{cm}$.
\end{abstract}

Keywords: CdTe; radiation detector; low pressure Bridgman method; chlorine doping

(C) Wroclaw University of Technology.

\section{Introduction}

CdTe is of considerable technological importance for use in semiconductor-based radiation detection applications, such as medical imaging and homeland security fields. The fabricated $\mathrm{CdTe}$ radiation detectors can be operated at room-temperature due to the favorable band gap $(1.44 \mathrm{eV})$ and have been researched by various groups [1-7].

High resistivity is required for any semiconductor material to be used in realizing highperformance radiation detectors. Because high bias voltage is needed to improve the charge collection efficiency of the detection devices, resistivity of the material used should be high enough, close to a semi-insulating state, since electronic noise of the material originating from the leakage current increases with larger amounts of free charge carriers within the semiconductor [8]. However, it has been reported that undoped CdTe bulk

\footnotetext{
*E-mail: jhha@kaeri.re.kr
}

crystal grown from the melt usually has p-type characteristics due to the acceptor-like nature of the inherent point defects including Cd-vacancies [9]. These point defects in the crystals can also behave as traps for increased leakage currents [10]. Also, another point defects such as Te inclusions have been known to adversely affect the charge transport properties, energy resolution, and uniformity of the fabricated radiation detectors [11-13]. Especially, Szeles et al. [13] discussed that the region of degraded charge transport near Te inclusions extends well beyond the volume of the inclusions themselves possibly due to the field of dislocations and Te precipitates surrounding Te inclusions. In this connection, $\mathrm{Cl}$ doping for annihilating all these point defects (i.e. Cd-vacancies and Te inclusions) can be an effective way to obtain semi-insulating (above $10^{9} \Omega \cdot \mathrm{cm}$ ) high-quality CdTe material [2, 8, 9, 13, 14].

Thus, in this study, we investigated the effects of $\mathrm{Cl}$ doping on the microstructural, electrical, and optical properties of CdTe ingots grown by using the low-pressure Bridgman (LPB) method. 
Samples from three different locations within each ingot (i.e. top, middle, bottom) were also compared. It was shown that the samples from the middle part of the ingot with $\mathrm{Cl}$ doping concentrations of $9.94 \times 10^{19} \mathrm{~cm}^{-3}$ and $1.99 \times 10^{20} \mathrm{~cm}^{-3}$ exhibited better crystalline qualities compared to other samples. It was also shown that the average size and surface coverage of Te inclusions decreased with increased $\mathrm{Cl}$ concentrations. Resistivity of the samples were increased by 5 to 6 orders of magnitude for Cl-doped samples. Optical properties of the samples measured by spectroscopic ellipsometry (SE) were also reported and discussed.

\section{Experimental}

\subsection{Sample preparation}

CdTe raw materials of $99.9999 \%$ purity were purchased from $5 \mathrm{~N}$ Plus Co. and were used asreceived. The powders were injected into 1-inch quartz ampoules which were sealed at a background pressure of $\sim 1.33 \times 10^{-4} \mathrm{~Pa}$. We put different amounts of $\mathrm{Cl}$ dopant into four different ampoules. In other words, one was undoped, and the other three were doped with $\mathrm{Cl}$ at the concentrations of $4.97 \times 10^{19} \mathrm{~cm}^{-3}, 9.94 \times 10^{19} \mathrm{~cm}^{-3}$ and $1.99 \times 10^{20} \mathrm{~cm}^{-3}$, respectively. The sealed ampoules were then loaded into a LPB furnace. For all four growth runs, 6-zone furnace moved at a speed of $0.93 \mathrm{~mm} / \mathrm{h}$ and the thermal gradient at the growth zone was approximately $10{ }^{\circ} \mathrm{C} / \mathrm{cm}$. Fig. 1 shows the four grown ingots used in this study. The ingots were then sliced perpendicular to the growth direction, by using a diamond wire saw. The samples from each part of the ingots (also designated in Fig. 1) are herein referred to as 'top', 'middle' and 'bottom' samples, respectively. Then, these samples were lapped with 800-, 2400- and 4000-grit $\mathrm{SiC}$ papers followed by polishing with different $\mathrm{Al}_{2} \mathrm{O}_{3}$ powders of $1 \mu \mathrm{m}, 0.3 \mu \mathrm{m}$ and $0.05 \mu \mathrm{m}$ diameters. To remove the mechanical damages at the sample surfaces during the processes, all the samples were chemically etched by dipping in the $2 \%$ $\mathrm{Br}$-methanol solutions. Finally, Au electrodes were deposited onto both sides of the samples by using the $\mathrm{AuCl}_{3}$ solutions.

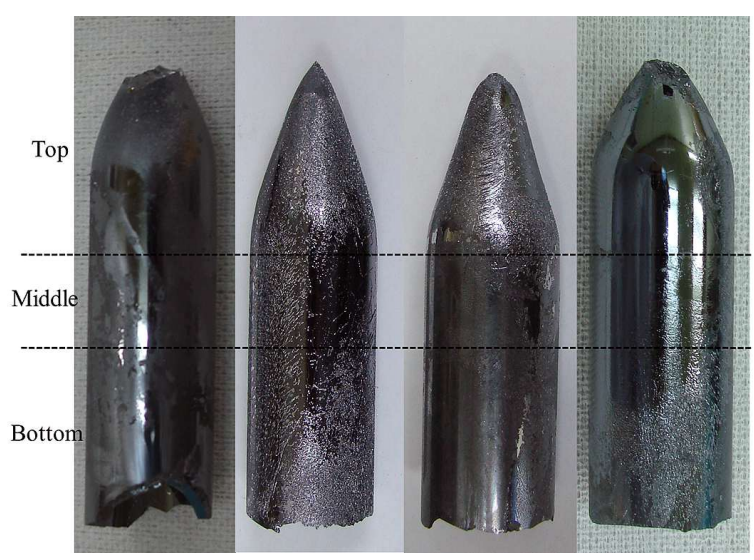

Fig. 1. Four CdTe ingots used in this study: undoped, doped with $\mathrm{Cl}$ at concentrations of $4.97 \times 10^{19} \mathrm{~cm}^{-3}, 9.94 \times 10^{19} \mathrm{~cm}^{-3}$ and $1.99 \times 10^{20} \mathrm{~cm}^{-3}$

\subsection{Characterization}

Microstructural perfections of the samples were investigated using an XRD (X-ray diffraction) system (D/Max 4200, Rigaku) within the 2 theta range of $20^{\circ}$ to $80^{\circ}$. Te inclusions within the samples were observed by infrared (IR) mapping system with a Nikon infrared camera. To characterize the optical properties of the samples, spectroscopic ellipsometry (SE, J.A. Woollam Co., photon energy: from $1.1 \mathrm{eV}$ to $6.5 \mathrm{eV}$ ) measurements at room temperature (RT) were performed. Finally, electrical measurements were performed by using a Keithley 4200 semiconductor characterization system.

\section{Results and discussion}

Fig. 2 shows XRD measurement results for the samples from four ingots with different $\mathrm{Cl}$ doping concentrations (undoped, $4.97 \times 10^{19} \mathrm{~cm}^{-3}$, $9.94 \times 10^{19} \mathrm{~cm}^{-3}$ and $1.99 \times 10^{20} \mathrm{~cm}^{-3}$ ) chosen from top, middle and bottom sections, respectively. Regardless of doping concentrations, samples from the middle section of the ingots exhibited better crystallinity, showing very sharp (1 1 1) peak. It is also shown that the middle samples with $\mathrm{Cl}$ doping concentrations of $9.94 \times 10^{19} \mathrm{~cm}^{-3}$ and $1.99 \times 10^{20} \mathrm{~cm}^{-3}$ exhibited slightly better crystal quality with reduced unwanted small peaks (e.g. (5 11 1) peak). 
(a) top

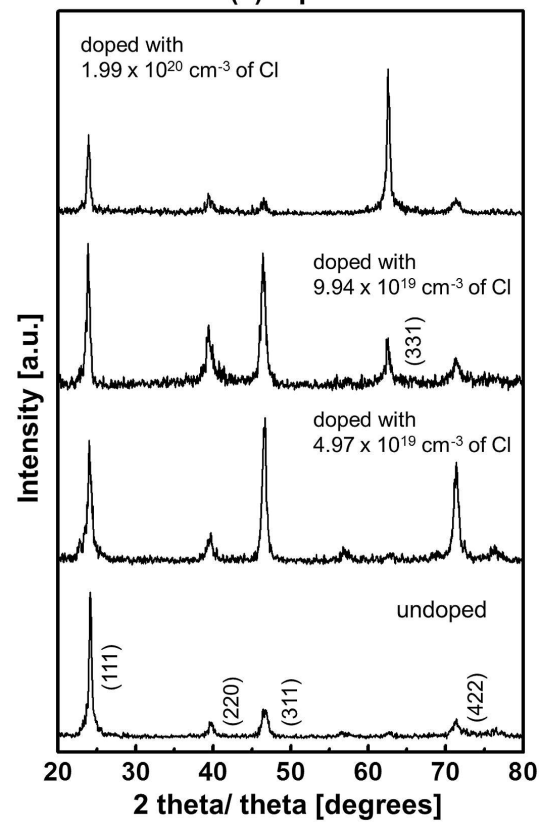

(b) middle

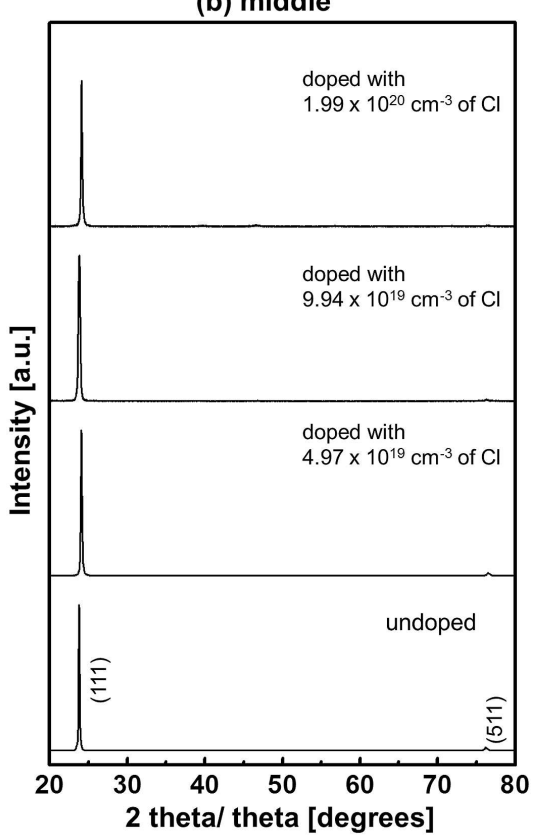

(c) bottom

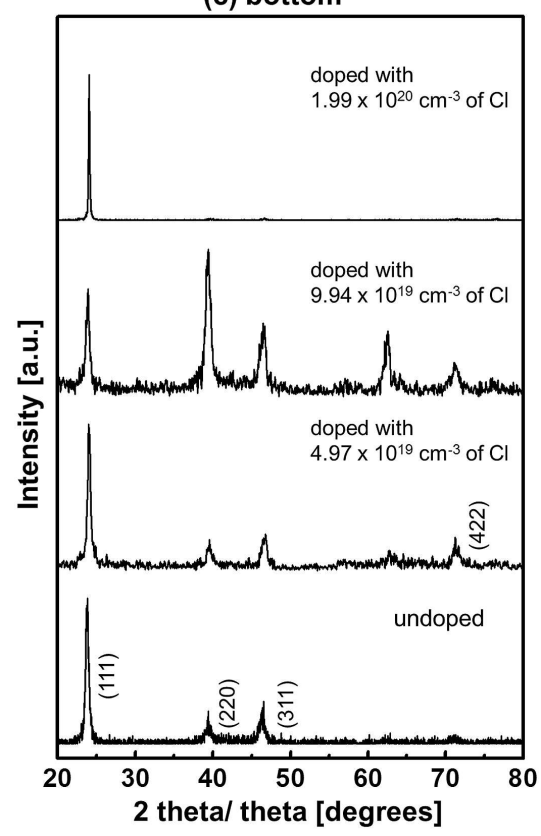

Fig. 2. XRD measurement results of 12 samples from four ingots with different $\mathrm{Cl}$ doping concentrations, chosen from top, middle and bottom sections, respectively.

Fig. 3 shows typical IR microscope images of analyzed from several IR microscope images Te inclusions in the middle samples with different (including Fig. 3) for the three samples are shown $\mathrm{Cl}$ doping concentrations $\left(4.97 \times 10^{19} \mathrm{~cm}^{-3}\right.$, in Fig. 4. It is noted from Fig. 3 and Fig. 4 $9.94 \times 10^{19} \mathrm{~cm}^{-3}$ and $1.99 \times 10^{20} \mathrm{~cm}^{-3}$ ). that the average size of the Te inclusions deAlso, histograms of the $\mathrm{Te}$ inclusion size creased with increasing $\mathrm{Cl}$ doping concentrations. 
For example, the samples with different $\mathrm{Cl}$ doping concentrations exhibited average sizes of $19.53 \mu \mathrm{m}$ $\left(4.97 \times 10^{19} \mathrm{~cm}^{-3}\right), 9.31 \mu \mathrm{m}\left(9.94 \times 10^{19} \mathrm{~cm}^{-3}\right)$ and $3.92 \mu \mathrm{m}\left(1.99 \times 10^{20} \mathrm{~cm}^{-3}\right)$. In addition, the observed average surface coverage of $\mathrm{Te}$ inclusions in the CdTe sample decreased with increasing $\mathrm{Cl}$ doping concentrations from $3.90 \%$ $\left(4.97 \times 10^{19} \mathrm{~cm}^{-3}\right), 1.86 \%\left(9.94 \times 10^{19} \mathrm{~cm}^{-3}\right)$ to $0.47 \%\left(1.99 \times 10^{20} \mathrm{~cm}^{-3}\right)$. Similar result was also obtained by Jayatirtha et al. [14]. However, it should be mentioned that the exact mechanisms underlying the participation of chlorine in the improvement of CdTe morphology are not clearly understood, what is also mentioned in the literature [9] and references therein, and the subject is currently under further investigations.

Fig. 5a shows a typical dielectric response of undoped and doped $\left(1.99 \times 10^{20} \mathrm{~cm}^{-3}\right)$ samples within the photon energy range of $1.1 \mathrm{eV}$ to $6.5 \mathrm{eV}$. From the data in Fig. 5a, extinction coefficient (k) and absorption coefficient $(\alpha=4 \pi \mathrm{k} / \lambda$, where $\lambda$ is wavelength in $\mathrm{cm}$ ) can be obtained. Because CdTe is a direct-transition semiconductor, its optical bandgap can be obtained by extrapolation of the linear part of the $\alpha^{2}$ vs. photon energy ( $h v$, in $\mathrm{eV}$ ) curves to $\alpha=0$ [15], as shown in Fig. 5b. It is noted that doped CdTe exhibits slightly larger bandgap, by $8 \mathrm{meV}$ compared to that of undoped sample, which means smaller Urbach tails below/above the conduction/valence band edges [16]. Fig. 5c shows the second derivative spectra [17] of the $\varepsilon_{1}$ curves (from Fig. 5a) near the bandgaps. Gamma values of the peaks [18] were obtained to be $0.169056 \mathrm{eV}$ for the undoped sample, and $0.153282 \mathrm{eV}$ for the doped sample. From Fig. 5b and Fig. 5c, the doped CdTe sample can be regarded to have better crystalline qualities.

Fig. 6a shows average resistivities of CdTe samples as a function of $\mathrm{Cl}$ doping concentration. It is shown that the resistivities have abruptly increased by almost 5 orders of magnitude with an addition of small amounts of $\mathrm{Cl}$ dopant $\left(4.97 \times 10^{19} \mathrm{~cm}^{-3}\right)$. Furthermore, with further increase in the dopant concentration, the resistivities were only slightly increased to about $10^{10} \Omega \cdot \mathrm{cm}$ as it is also summarized in Table 1. Similar resistivity saturation

\section{(a) doped with $4.97 \times 10^{19} \mathrm{~cm}^{-3}$ of $\mathrm{Cl}$}

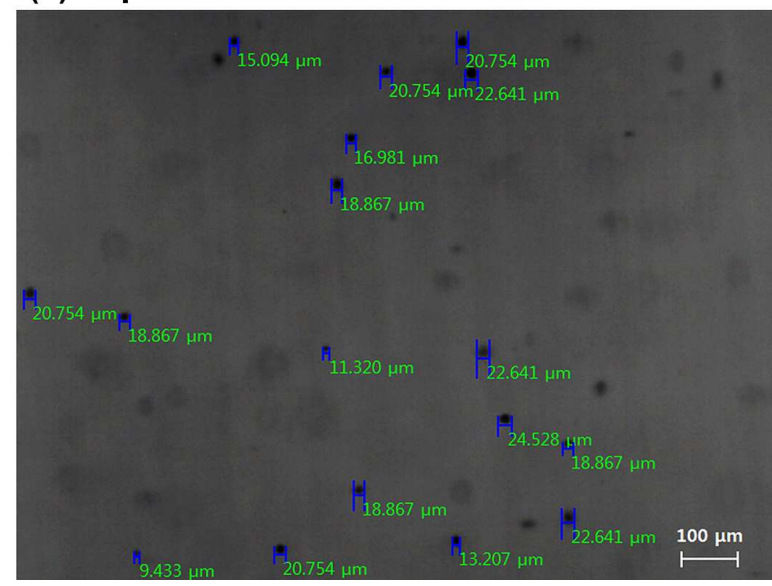

(b) doped with $9.94 \times 10^{19} \mathrm{~cm}^{-3}$ of $\mathrm{Cl}$

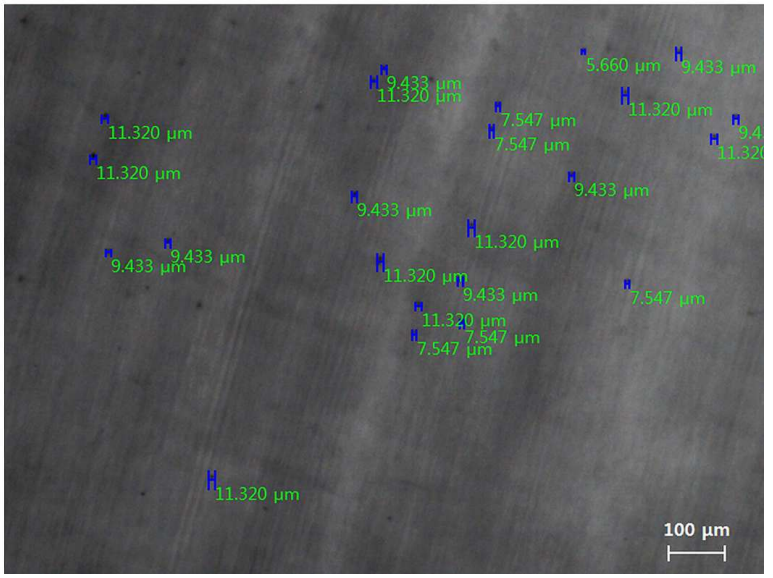

(c) doped with $1.99 \times 10^{20} \mathrm{~cm}^{-3}$ of $\mathrm{Cl}$

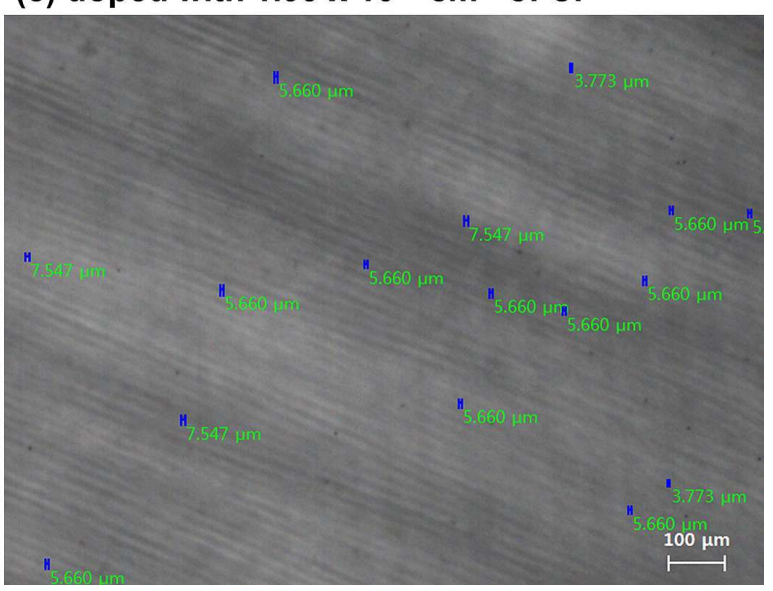

Fig. 3. Typical IR microscope images of Te inclusions for three samples with $\mathrm{Cl}$ doping concentrations of $4.97 \times 10^{19} \mathrm{~cm}^{-3}, 9.94 \times 10^{19} \mathrm{~cm}^{-3}$ and $1.99 \times 10^{20} \mathrm{~cm}^{-3}$. The solid lines appearing on the surfaces might be due to the scratches induced by incompletely optimized polishing processes. 

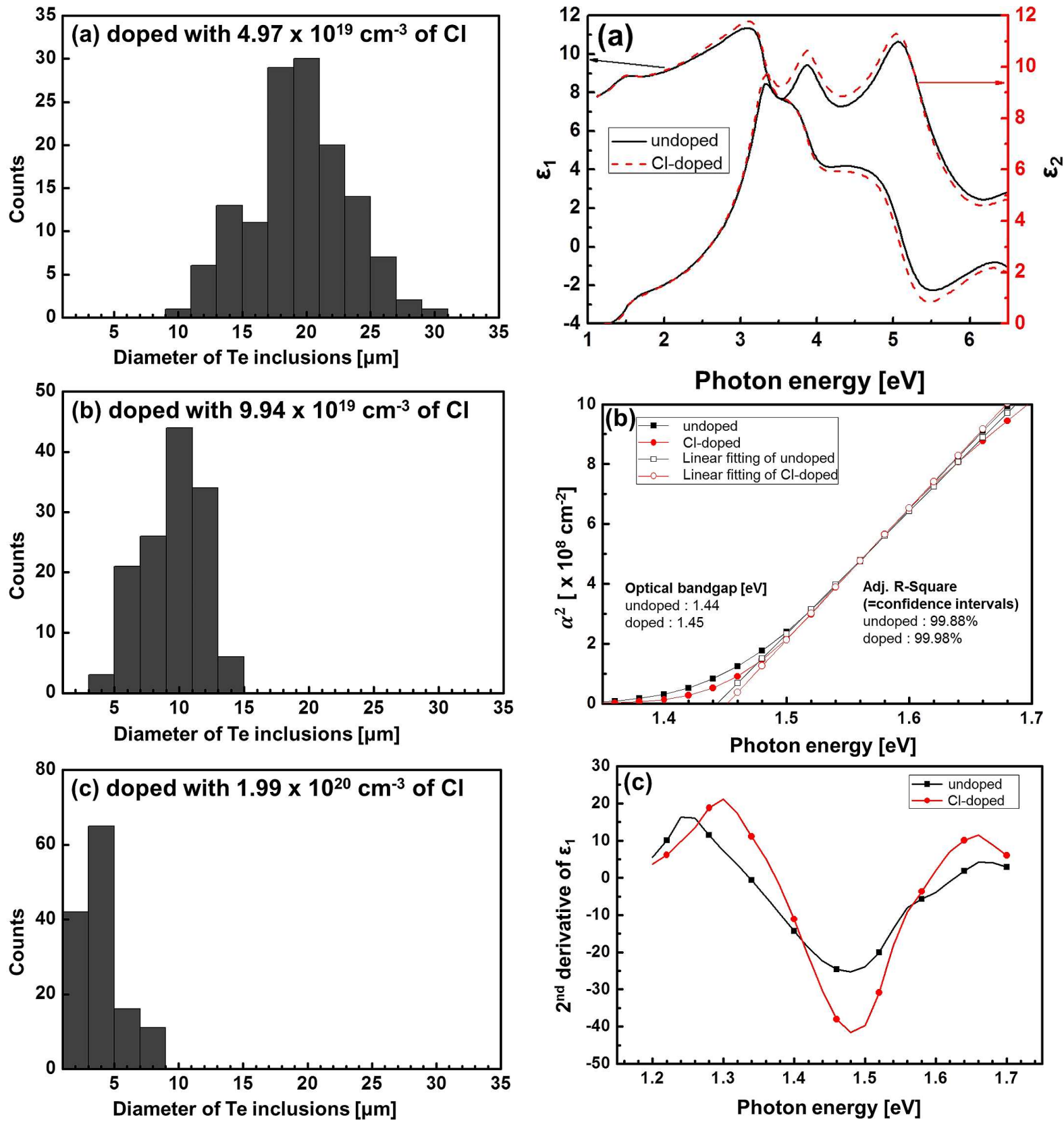

Fig. 4. Histogram of Te inclusions size distribution for three Cl-doped CdTe samples.

phenomenon was also observed by Shin et al. [19]. Fig. 6b shows variation of average resistivities of the samples as a function of different locations within the ingots. It is shown that the samples from the middle part of the ingots exhibit slightly higher resistivities compared to the other parts (i.e. top or bottom samples), as has been also

Fig. 5. (a) Dielectric response curves of undoped and doped $\left(1.99 \times 10^{20} \mathrm{~cm}^{-3}\right)$ CdTe sample, (b) $\alpha^{2}$ vs. photon energy $(\mathrm{h} v$, in $\mathrm{eV})$ with extrapolation of the linear part of the curves to $\alpha=0$, (c) second derivative plots of $\varepsilon_{1}$ shown in Fig. 5a.

summarized in Table 1. This might be due to the fact that the samples with poorer crystalline quality have higher density of unwanted parasitic shunt paths for charge carriers [20] leading to lower resistivities. 
Table 1. Average resistivities of the CdTe samples with different $\mathrm{Cl}$ doping concentrations and different positions within the ingots. Mean values for each row/column were also calculated and presented in the Table.

\begin{tabular}{ccccc}
\hline & Top $[\Omega \cdot \mathrm{cm}]$ & Middle $[\Omega \cdot \mathrm{cm}]$ & Bottom $[\Omega \cdot \mathrm{cm}]$ & Mean $[\Omega \cdot \mathrm{cm}]$ \\
\hline \hline Undoped & $5.20 \times 10^{4}$ & $1.33 \times 10^{4}$ & $1.60 \times 10^{4}$ & $2.71 \times 10^{4}$ \\
$\mathrm{Cl}$ doped, $4.97 \times 10^{19} \mathrm{~cm}^{-3}$ & $3.56 \times 10^{6}$ & $5.70 \times 10^{9}$ & $2.07 \times 10^{9}$ & $2.59 \times 10^{9}$ \\
$\mathrm{Cl}$ doped, $9.94 \times 10^{19} \mathrm{~cm}^{-3}$ & $1.06 \times 10^{9}$ & $8.03 \times 10^{9}$ & $3.35 \times 10^{9}$ & $4.15 \times 10^{9}$ \\
Cl doped, $1.99 \times 10^{20} \mathrm{~cm}^{-3}$ & $2.19 \times 10^{9}$ & $1.44 \times 10^{10}$ & $2.11 \times 10^{9}$ & $6.22 \times 10^{9}$ \\
Mean $[\Omega \cdot \mathrm{cm}]$ & $8.15 \times 10^{8}$ & $7.02 \times 10^{9}$ & $1.88 \times 10^{9}$ & \\
\hline
\end{tabular}
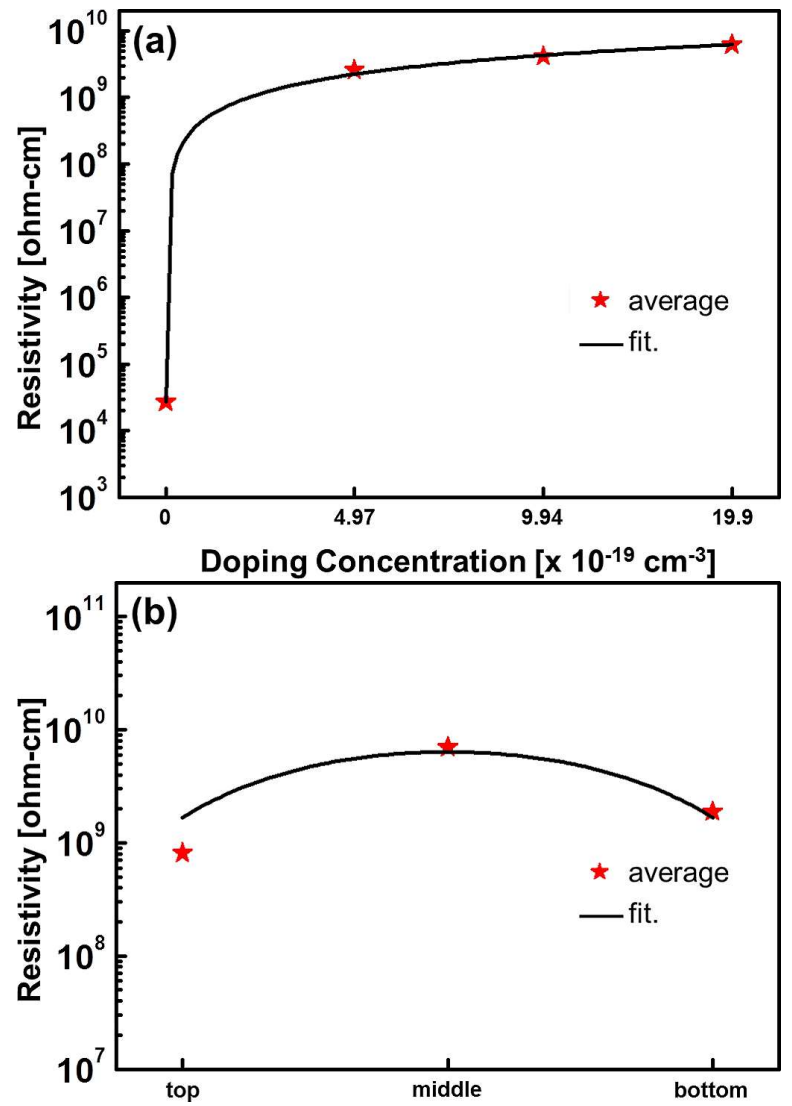

Fig. 6. (a) Average resistivities of CdTe samples as a function of $\mathrm{Cl}$ doping concentrations. Solid fitting line was provided as a guide for the eyes; (b) average resistivities of CdTe samples from different locations within the ingots (Fig. 1). Solid fitting line was provided as a guide for the eyes.

Although the exact mechanisms of the role of $\mathrm{Cl}$ dopant during the $\mathrm{CdTe}$ growth are not clearly understood at this moment, the following can be stated. Based on the structure-property relationships, $\mathrm{Cl}$ dopant in $\mathrm{CdTe}$ may take an important role to improve the crystalline quality of CdTe. In other words, XRD measurement results (Fig. 2) show that the doped samples exhibit reduced, thus almost negligible $\left(\begin{array}{lll}5 & 1 & 1\end{array}\right)$ peaks. Our preliminary XRF (X-ray fluorescence) measurements results for the undoped and doped $\left(1.99 \times 10^{20} \mathrm{~cm}^{-3}\right)$ samples (not shown) also showed that the $\mathrm{Cl}$ dopants were clearly observed and properly incorporated within the host CdTe material. In this work, it has been shown that $\mathrm{Cl}$ dopant helped reduce Te inclusions as shown in Fig. 3 and Fig. 4. In addition, effects of $\mathrm{Cl}$ dopant in enhancing the CdTe material quality from the optical point of view are observed in Fig. 5. In other words, the optical bandgap and gamma value are larger and smaller, respectively, for doped samples as can be observed in Fig. 5. Finally, from resistivity calculations, $\mathrm{Cl}$ dopant was shown to successfully compensate native point defects, resulting in the semiinsulating CdTe, as shown in Fig. 6. Thus, although the optimum doping concentrations were reported by different research groups to be slightly different $[9,13,19]$, we can assume that $\mathrm{Cl}$ doping is an effective way to enhance the material properties, which can be beneficial for making highperformance radiation detectors.

\section{Conclusions}

CdTe crystals doped with different amounts of $\mathrm{Cl}$ were characterized for use in radiation detection applications. It was shown that the CdTe crystals from the middle parts of the ingots doped at concentrations of $9.94 \times 10^{19} \mathrm{~cm}^{-3}$ and $1.99 \times 10^{20} \mathrm{~cm}^{-3}$ exhibited better crystalline qualities as observed from XRD, IR mapping, 
and SE measurement and analysis results. It was further observed that the CdTe samples doped with a doping concentration of $1.99 \times 10^{20} \mathrm{~cm}^{-3}$ from the middle part of the ingot exhibited an average resistivity of $1.44 \times 10^{10} \Omega \cdot \mathrm{cm}$. These findings clearly demonstrate that our Cl-doped CdTe samples can successfully be used for fabricating highperformance radiation detectors.

\section{Acknowledgements}

We are grateful to Prof. Tae Jung Kim and Prof. Young Dong Kim, Mr. Han Gyeol Park (all with the Kyung Hee University) for fruitful discussions regarding SE analysis. The authors would like to gratefully acknowledge the financial support by the Nuclear R\&D Programs of the Ministry of Science, the ICT \& Future Planning (MSIP) of South Korea (NRF2010-0026096, NRF-2013M2A2A4023359), a grant from the Korea Atomic Energy Research Institute (Grant No.: 52328015), and by the WCU (World Class University) Program through the National Research Foundation (NRF) of Korea funded by the Ministry of Education, Science and Technology (R31-2008-10029).

\section{References}

[1] Audet N., Guskov V.N., Greenberg J.H., J. Electron. Mater., 34 (2005), 687.

[2] Shiraki H., Funaki M., Ando Y., Tachibana A., Kominami S., OHNo R., IEEE T. Nucl. Sci., 56 (2009), 1717.

[3] Saminadayar K., Galland D., Molv E., Solid State Commun., 49 (1984), 627.

[4] Meyer B.K., HofmanN D.M., Stadler W., SAlK M., EICHE C., BEnZ K.W., Proceedings of the MRS Spring Meeting, San Francisco, 1993.

[5] Weigel E., Milller-Vogt G., Steinbach B., Wendl W., Stadler W., Hofmann D.M., Meyer B.K., Mater. Sci. Eng. B-Adv., 16 (1993), 17.
[6] Korbutyak D.V., Krylyuk S.G., Tkachuk P.M., TKACHUK V.I., KORBUTJAK N.D., RARANSKY M.D., J. Cryst. Growth, 197 (1999), 662.

[7] Fiederle M., Babentsov V., FranC J., Fauler A., Konrath J.-P., Cryst. Res. Technol., 38 (2003), 588.

[8] Matveev O.A., Terentev A.I., Semiconductors, 34 (2000), 1264.

[9] Popovych V.D., Virt I.S., Sizov F.F., TEtyorkin V.V., TsybriI(IVAsiv) Z.F., DARChuk L.O., PARFENJUK O.A., IlASHCHUK M.I., J. Cryst. Growth, 308 (2007), 63.

[10] Sochinskit N.V., Lozano M., Pellegrini G., UlLAN M., Nucl. Instrum. Meth. A, 568 (2006), 451.

[11] Funaki M., Ozaki T., Satoh K., Ohno R., Nucl. Instrum. Meth. A, 436 (1999), 120.

[12] Bolotnikov A.E., Camarda G.S., Carini G.A., Cui Y., Kohman K.T., Li L., Salomon M.B., JAMES R.B., IEEE T. Nucl. Sci., 54 (2007), 821.

[13] Szeles C., Cameron S.E., Soldner S.A., NdaP J.-O., REED M.D., J. Electron. Mater., 33 (2004), 742.

[14] Jayatirtha H.N., Henderson D.O., Burger A., Volz M.P., Appl. Phys. Lett., 62 (1993), 573.

[15] Oн J.-H., Kim K.-K., SeOng T.-Y., Appl. Surf. Sci., 257 (2011), 2731.

[16] MaSnAdi-Shirazi M., LEWIS R.B., BAhramiYekta V., Tiedje T., Chicoine M., Servati P., J. Appl. Phys., 116 (2014), 223506.

[17] Lautenschlager P., Garriga M., LogotheTIDIS S., CARDona M., Phys. Rev. B, 35 (1987), 9174.

[18] Arwin H., Aspnes D.E., J. Vac. Sci. Technol. A, 2 (1984), 1316.

[19] ShIn H.-Y., Sun C.-Y., J. Cryst. Growth, 186 (1998), 67.

[20] VAn Dyk E.E., Meyer E.L., Renew. Energ., 29 (2004), 333.

Received 2015-06-17

Accepted 2016-05-17 